\title{
An Expansion Based on Sine-Gordon Equation to Solve KdV and modified KdV Equations in Conformable Fractional Forms
}

\author{
Ozlem Ersoy Hepson ${ }^{a}$, Alper Korkmaz $^{b, *}$ Kamyar Hosseini ${ }^{c}$, \\ Hadi Rezazadeh ${ }^{d}$, Mostafa Eslami ${ }^{e}$ \\ ${ }^{a}$ Eskişehir Osmangazi University, Department of Computer and Mathematics, Eskişehir, Turkey. \\ ${ }^{b}$ Çankırı Karatekin University, Department of Mathematics, 18200, Çankırı, Turkey. \\ ${ }^{c}$ Department of Mathematics, Rasht Branch, Islamic Azad University, Rasht, Iran. \\ ${ }^{d}$ Faculty of Engineering Technology, Amol University of Special Modern Technologies, Amol, Iran. \\ ${ }^{e}$ Department of Mathematics, Faculty of Mathematical Sciences, University of Mazandaran, Babolsar, Iran.
}

\begin{abstract}
An expansion method based on time fractional Sine-Gordon equation is implemented to construct some real and complex valued exact solutions to the Korteweg-de Vries and modified Korteweg-de Vries equation in time fractional forms. Compatible fractional traveling wave transform plays a key role to be able to apply homogeneous balance technique to set the predicted solution. The relation between trigonometric and hyperbolic functions based on fractional Sine-Gordon equation allows to form the exact solutions with multiplication of powers of hyperbolic functions.
\end{abstract}

Keywords: Sine-Gordon Expansion Method; Conformable time fractional KdV Equation; Conformable time fractional modified KdV Equation; Exact Solution, Traveling Wave Solution.

MSC2010: 35C07;35R11;35Q53.

PACS: 02.30.Jr; 02.70.Wz; 04.20.Jb

*alperkorkmaz7@gmail.com 


\section{Introduction}

Probably the most famous and fundamental weakly nonlinear PDE with soliton type wave solutions in shallow water surface is Korteweg-de Vries (KdV) equation. Even though it was firstly introduced by Boussinesq [1] in 1877, it is named after the study of Korteweg and de Vries [2]. The KdV equation is completely integrable and has wave solutions of classical solitary wave type shapes. Moreover, it has infinitely many conservation laws representing various physical quantities such as mass, momentum, energy, etc. preserved during motion [3]. The equation can describe many types of physical phenomena particularly waves covering internal ocean waves in changing density layers, plasma ion-acoustic waves and acoustictype waves over crystal lattice.

The KdV equation is similar to nonlinear Schrödinger equation due to the fact that both are solvable by inverse scattering transform approach. It has stable N-soliton solutions that behave like particles, too [4]. These solutions are valid for multiple collisions,i.e. more than two well-separated solitons, even when their heights are different from each other [5].

Recent developments in computer algebra lead various solution techniques to appear. Different forms of tanh - coth method was implemented to the KdV equation to determine periodic and soliton type wave solutions $[6] .\left(G^{\prime} / G\right)$-expansion method is also capable of setting the exact solutions of the KdV equation in various rational forms of hyperbolic or trigonometric function series [7]. Some periodic solitary type wave solutions are determined by extended form of homoclinic test method [8].

The modified form of the $\mathrm{KdV}(\mathrm{mKdV})$ equation is obtained by changing the nonlinear term $u u_{z}$ to $u^{2} u_{z}$ in the $\mathrm{KdV}$ equation. Wronskian expansion technique was used to determine composite function solutions to the mKdV equation [9]. Implementation of simple sin - cos ansatz techniques gives some traveling type wave solutions in forms of trigonometric and hyperbolic functions [10]. The exp function approach also determines some periodic solutions including the rational functions of exponential and trigonometric function in both numerator and denominator [11]. In this study, we focus on the conformable time fractional forms of the KdV equation

$$
D_{t}^{\gamma} u+p u u_{z}+q u_{z z z}=0, t>0, \gamma \in(0,1]
$$

and the modified $\mathrm{KdV}$ equation

$$
D_{t}^{\gamma} u+p u^{2} u_{z}+q u_{z z z}=0, t>0, \gamma \in(0,1]
$$

where $u$ is function of the independent variables $t$ and $z, p$ and $q$ are real parameters. The operator $D_{t}^{\gamma}$ represents conformal fractional derivative operator defined only for positive region of $t$ [12]. Different from some classical methods 
such as various forms of Kudryashov approach, exponential rational function technique, simple hyperbolic ansatzes [13-22], the fractional form of the Sine-Gordon equation method is implemented to both equations to derive exact solutions in traveling wave forms. Before constructing the solutions, some preliminaries and basic properties of conformable derivative are given below. A brief summary of the method is also given in the next sections.

\section{Preliminaries of Conformable Derivative}

$\gamma$.th order conformable derivative of a conformably differentiable function $T=T(t)$ is defined as

$$
D_{t}^{\gamma}(T(t))=\lim _{\epsilon \rightarrow 0} \frac{T\left(t+\epsilon t^{1-\gamma}\right)-T(t)}{\tau}, t>0, \gamma \in(0,1]
$$

where $T=T(t):[0, \infty) \rightarrow \mathbb{R}[12]$. This form of the fractional derivative satisfies many properties required to be able to study to solve non linear fractional PDEs. Some of these properties can be summarized in the following theorem.

Theorem 1 Let $S=S(t)$ and $T=T(t)$ are $\gamma$-differentiable in the positive part of t-axis. Then,

- $D_{t}^{\gamma}\left(k_{1} S(t)+k_{2} T(t)\right)=k_{1} D_{t}^{\gamma}(S(t))+k_{2} D_{t}^{\gamma}(T(t))$

- $D_{t}^{\gamma}\left(t^{m}\right)=m t^{m-\gamma}, \forall m \in \mathbb{R}$

- $D_{t}^{\gamma}\left(k_{3}\right)=0$, for all constant functions $S(t)=k_{3}$

- $D_{t}^{\gamma}(S(t) T(t))=S(t) D_{t}^{\gamma}(T(t))+T(t) D_{t}^{\gamma}(S(t))$

- $D_{t}^{\gamma}\left(\frac{S(t)}{T(t)}\right)=\frac{T(t) D_{t}^{\gamma}(S(t))-S(t) D_{t}^{\gamma}(T(t))}{T^{2}(t)}$

- $D_{t}^{\gamma}(S(t))=t^{1-\gamma} \frac{d S(t)}{d t}$

for $\forall k_{1}, k_{2}, k_{3} \in \mathbb{R}$ [23].

This newly introduced fractional derivative satisfies plenty of helpful properties. Some of these properties like the chain rule, some integral transforms, and Taylor series expansion were summarized in [24]. The following theorem gives the relation between the classical and conformable derivative.

Theorem 2 Let $S=S(t)$ be a $\gamma$-conformable differentiable function. Then,

$$
D_{t}^{\gamma}(S \circ T)(t)=t^{1-\gamma} T^{\prime}(t) S^{\prime}(T(t))
$$

where $\circ$ denotes composite function, the differentiable function $T=T(t)$ is defined in the range of $S(t)$. 


\section{Sine-Gordon Equation (SGE) Method}

Consider the one dimensional time fractional SGE of the form

$$
\frac{\partial^{2} u}{\partial z^{2}}-D_{t}^{2 \gamma} u=c^{2} \sin u, \quad c \text { is constant }
$$

where $u=u(z, t)$. The fractional traveling wave transform

$$
\begin{array}{r}
u(z, t) \rightarrow U(\eta) \\
\eta=a\left(z-\nu t^{\gamma} / \gamma\right)
\end{array}
$$

reduces the time fractional SGE (5) to

$$
\frac{d^{2} U}{d \eta^{2}}=\frac{m^{2}}{a^{2}\left(1-\nu^{2}\right)} \sin U
$$

where $\nu$ is speed parameter of the wave defined in the fractional traveling wave transform [25]. After some calculus, the equation is reduced to

$$
\left(\frac{d(U / 2)}{d \eta}\right)^{2}=\frac{m^{2}}{a^{2}\left(1-\nu^{2}\right)} \sin ^{2} U / 2+C
$$

where $C$ is integration constant and assumed zero. Assume that $w(\eta)=U(\eta) / 2$ and $m^{2} /\left(a^{2}\left(1-\nu^{2}\right)\right)=1$. Then, (8) takes the form

$$
\frac{d(w)}{d \eta}=\sin w
$$

Thus, (9) gives the following relations

$$
\sin w(\eta)=\left.\frac{2 c e^{\eta}}{c^{2} e^{2 \eta}+1}\right|_{c=1}=\operatorname{sech} \eta
$$

or

$$
\cos w(\eta)=\left.\frac{c^{2} e^{2 \eta}-1}{c^{2} e^{2 \eta}+1}\right|_{c=1}=\tanh \eta
$$

where $c \neq 0$ is integral constant.

On the other hand, the governing fractional PDE

$$
\Omega\left(u, D_{t}^{\gamma} u, u_{z}, D_{t t}^{2 \gamma} u, u_{z z}, \ldots\right)=0
$$

is reduced to an ODE of the form

$$
\tilde{\Omega}\left(U, U^{\prime}, U^{\prime \prime}, \ldots\right)=0
$$


by using the fractional traveling wave transform $u(z, t)=U(\eta)$ with the transform variable $\eta=a\left(z-\nu t^{\gamma} / \gamma\right)$. Then, a predicted solution to (13) of the form

$$
U(\eta)=A_{0}+\sum_{i=1}^{s} \tanh ^{i-1}(\eta)\left(B_{i} \operatorname{sech} \eta+A_{i} \tanh \eta\right)
$$

is constructed. This solution can be expressed in terms of $w$ as

$$
U(w)=A_{0}+\sum_{i=1}^{s} \cos ^{i-1}(w)\left(B_{i} \sin w+A_{i} \cos w\right)
$$

due to the relations between hyperbolic and trigonometric functions given (10) (11). The solution procedure starts by determining the degree $s$ of the predicted power series solution of trigonometric functions. The balance between the non linear term and the derivative term with highest order is the key to find $s$. Once it is determined, the predicted solution can be written as a finite power series of multiplication of trigonometric functions. Substitution of the predicted solution (15) into (13) is followed by equating the coefficients of powers of $\sin w \cos w$ to zero. The resultant algebraic system is solved for the coefficients $A_{0}, A_{1}, B_{1}, \ldots$ and at least one of transform non zero coefficients $a$ and $\nu$. Later on, the determined parameters are substituted in to the predicted solution (15). Changing the trigonometric functions to hyperbolic functions by using (10) - (11) gives the next form of the solution. Finally, the solutions are expressed in original variables $z$ and $t$. $a$ and $\nu$ are also substituted into the final forms of the solutions.

\section{Solutions to the conformable time fractional KdV Equation}

The KdV equation (1) reads

$$
-a \nu U^{\prime}+a p U U^{\prime}+q a^{3} U^{\prime \prime \prime}=0
$$

under the fractional traveling wave transform (6). The balance number is calculated as 2 when $U U^{\prime}$ and $U^{\prime \prime \prime}$ are considered. Thus, the predicted solution takes the form

$$
U(w)=A_{0}+B_{1} \sin (w)+A_{1} \cos (w)+\cos (w) B_{2} \sin (w)+A_{2}(\cos (w))^{2}
$$


Substituting the predicted solution (17) into (16) gives

$$
\begin{aligned}
& a^{3} q B_{2} \sin (w(\eta))(\cos (w(\eta)))^{4}+\left(-8 a^{3} q A_{2}-2 a p A_{2}^{2}+a p B_{2}^{2}\right)(\sin (w(\eta)))^{2}(\cos (w(\eta)))^{3} \\
& +\left(a^{3} q B_{1}+a p A_{1} B_{2}+a p A_{2} B_{1}\right) \sin (w(\eta))(\cos (w(\eta)))^{3} \\
& +\left(-18 a^{3} q B_{2}-4 a p A_{2} B_{2}\right)(\sin (w(\eta)))^{3}(\cos (w(\eta)))^{2} \\
& +\left(-4 a^{3} q A_{1}-3 a p A_{1} A_{2}+2 a p B_{1} B_{2}\right)(\sin (w(\eta)))^{2}(\cos (w(\eta)))^{2} \\
& +\left(a p A_{0} B_{2}+a p A_{1} B_{1}-a \nu B_{2}\right) \sin (w(\eta))(\cos (w(\eta)))^{2} \\
& +\left(16 a^{3} q A_{2}-a p B_{2}{ }^{2}\right)(\sin (w(\eta)))^{4} \cos (w(\eta)) \\
& +\left(-5 a^{3} q B_{1}-2 a p A_{1} B_{2}-2 a p A_{2} B_{1}\right)(\sin (w(\eta)))^{3} \cos (w(\eta)) \\
& +\left(-2 a p A_{0} A_{2}-a p A_{1}{ }^{2}+a p B_{1}{ }^{2}+2 a \nu A_{2}\right)(\sin (w(\eta)))^{2} \cos (w(\eta)) \\
& +\left(a p A_{0} B_{1}-a \nu B_{1}\right) \sin (w(\eta)) \cos (w(\eta))+5(\sin (w(\eta)))^{5} a^{3} q B_{2} \\
& +\left(2 a^{3} q A_{1}-a p B_{1} B_{2}\right)(\sin (w(\eta)))^{4}+\left(-a p A_{0} B_{2}-a p A_{1} B_{1}-a p A_{2} B_{2}+a \nu B_{2}\right)(\sin (w(\eta)))^{3} \\
& +\left(-a p A_{0} A_{1}+a \nu A_{1}\right)(\sin (w(\eta)))^{2}+a p A_{2} B_{2} \sin (w(\eta))=0
\end{aligned}
$$

Following some algebra and substitution of some trigonometric identities and rearrange of coefficients of the terms, we assume that all coefficients are zero. Thus, we find the system

$$
\begin{array}{r}
-3 a\left(2 a^{2} q A_{1}+p A_{1} A_{2}-p B_{1} B_{2}\right)+8 a^{3} q A_{1}-a p A_{0} A_{1}+3 a p A_{1} A_{2}-4 a p B_{1} B_{2}+a \nu A_{1}=0 \\
5 a^{3} q B_{2}-a p A_{0} B_{2}-a p A_{1} B_{1}+a \nu B_{2}=0 \\
24 a^{3} q B_{2}+4 a p A_{2} B_{2}=0 \\
6 a^{3} q B_{1}+3 a p A_{1} B_{2}+3 a p A_{2} B_{1}=0 \\
-28 a^{3} q B_{2}+2 a p A_{0} B_{2}+2 a p A_{1} B_{1}-3 a p A_{2} B_{2}-2 a \nu B_{2}=0 \\
-5 a^{3} q B_{1}+a p A_{0} B_{1}-2 a p A_{1} B_{2}-2 a p A_{2} B_{1}-a \nu B_{1}=0 \\
a\left(24 a^{2} q A_{2}+2 p A_{2}{ }^{2}-2 p B_{2}{ }^{2}\right)=0 \\
6 a^{3} q A_{1}+3 a p A_{1} A_{2}-3 a p B_{1} B_{2}=0 \\
a\left(-16 a^{2} q A_{2}+2 p A_{0} A_{2}+p A_{1}{ }^{2}-p B_{1}{ }^{2}+p B_{2}{ }^{2}-2 \nu A_{2}\right)-a\left(24 a^{2} q A_{2}+2 p A_{2}{ }^{2}-2 p B_{2}{ }^{2}\right)=0 \\
-14 a^{3} q A_{1}+a p A_{0} A_{1}-6 a p A_{1} A_{2}+7 a p B_{1} B_{2}-a \nu A_{1}+3 a\left(2 a^{2} q A_{1}+p A_{1} A_{2}-p B_{1} B_{2}\right)=0 \\
-a\left(-16 a^{2} q A_{2}+2 p A_{0} A_{2}+p A_{1}{ }^{2}-p B_{1}{ }^{2}+p B_{2}{ }^{2}-2 \nu A_{2}\right)=0
\end{array}
$$


Solution of this algebraic system for $\nu, A_{0}, A_{1}, A_{2}, B_{1}$ and $B_{2}$ gives

$$
\begin{aligned}
& \nu=-8 a^{2} q+p A_{0}, A_{1}=0, A_{2}=-\frac{12 a^{2} q}{p}, B_{1}=0, B_{2}= \\
& \nu=-5 a^{2} q+p A_{0}, A_{1}=0, A_{2}=-\frac{6 a^{2} q}{p}, B_{1}=0, B_{2}=\frac{6 a^{2} q}{p} i \\
& \nu=-5 a^{2} q+p A_{0}, A_{1}=0, A_{2}=-\frac{6 a^{2} q}{p}, B_{1}=0, B_{2}=-\frac{6 a^{2} q}{p} i
\end{aligned}
$$

where $a, A_{0}$ arbitrarily chosen constants and $i=\sqrt{-1}$. The real solution of the conformable $\mathrm{KdV}$ equation is expressed as

$$
u_{1}(z, t)=A_{0}-\frac{12 a^{2} q}{p} \tanh ^{2}\left(a\left(z+\left(8 a^{2} q-p A_{0}\right) \frac{t^{\gamma}}{\gamma}\right)\right)
$$

for arbitrary $a \neq 0$ and $A_{0}$. On the other hand, the complex solutions are represented as

$$
\begin{aligned}
u_{2,3}(z, t) & =A_{0}-\frac{6 a^{2} q}{p} \tanh ^{2}\left(a\left(z+\left(5 a^{2} q-p A_{0}\right) \frac{t^{\gamma}}{\gamma}\right)\right) \\
& \pm \frac{6 a^{2} q}{p} \tanh \left(a\left(z+\left(5 a^{2} q-p A_{0}\right) \frac{t^{\gamma}}{\gamma}\right)\right) \operatorname{sech}\left(a\left(z+\left(5 a^{2} q-p A_{0}\right) \frac{t^{\gamma}}{\gamma}\right)\right)
\end{aligned}
$$

The real valued exact solution is pictured out in Fig 1(a)-1(d) for various derivative orders in a finite domain of $z$ and $t$. This solution is a particular form of $u_{1}(z, t)$ determined by assuming $A_{0}=1, a=-1, q=-1 / 2, p=1$. These parameters gives a solution with upside-down pulse whose nadir is located at the origin initially. Parallel to proceeding time, the initial wave with negative amplitude moves along the positive $z$-direction by preserving its shape and depth. Even though the velocity of the pulse changes for smaller values of $\gamma$, we observe a linear motion with constant speed for $\gamma=1$, Fig $1(\mathrm{~d})$. 


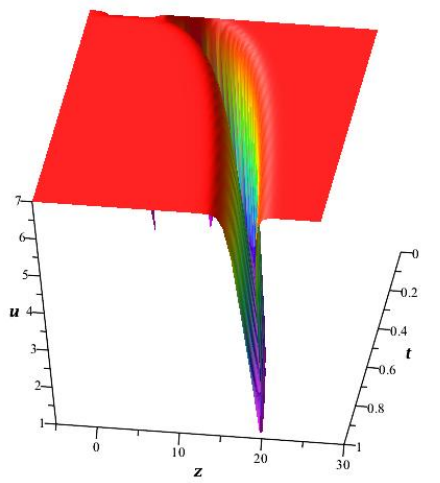

(a) $\gamma=0.25$

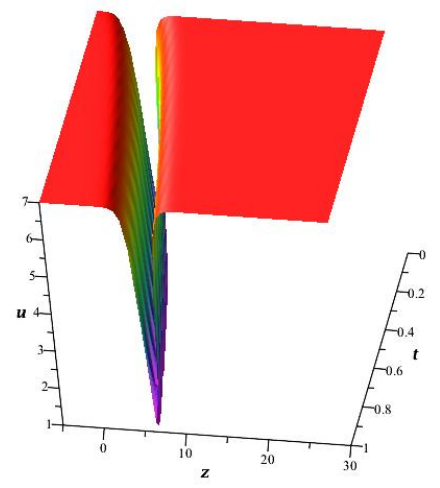

(c) $\gamma=0.75$

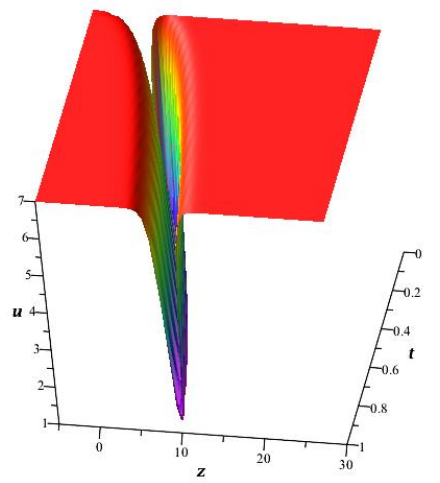

(b) $\gamma=0.50$

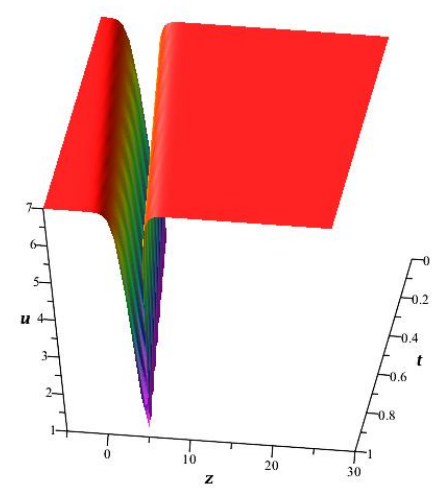

(d) $\gamma=1$

Figure 1: The solution $u_{1}(z, t)=1+6 \tanh ^{2}\left(-z+\frac{5 t^{\gamma}}{\gamma}\right)$ for various derivative orders

\section{Solutions to the conformable time fractional mKdV Equation}

The fractional traveling wave transform (6) reduces the mKdV equation (2) into

$$
-a \nu U^{\prime}+a p U^{2} U^{\prime}+q a^{3} U^{\prime \prime \prime}=0
$$

where ' denotes the classical derivative with respect to $\eta . s$ is determined as 1 by balancing $U^{2} U^{\prime}$ and $U^{\prime \prime \prime}$. Thus, the predicted solution is set as

$$
U(w)=A_{0}+B_{1} \sin (w)+A_{1} \cos (w)
$$


Substitution of this solution into (23) reads

$$
\begin{aligned}
& \left(a^{3} q B_{1}+a p A_{1}^{2} B_{1}\right) \sin (w(\eta))(\cos (w(\eta)))^{3} \\
& +\left(-4 a^{3} q A_{1}-a p A_{1}^{3}+2 a p A_{1} B_{1}^{2}\right)(\sin (w(\eta)))^{2}(\cos (w(\eta)))^{2} \\
& +2(\cos (w(\eta)))^{2} a p A_{0} A_{1} B_{1} \sin (w(\eta)) \\
& +\left(-5 a^{3} q B_{1}-2 a p A_{1}^{2} B_{1}+a p B_{1}^{3}\right)(\sin (w(\eta)))^{3} \cos (w(\eta)) \\
& +\left(-2 a p A_{0} A_{1}^{2}+2 a p A_{0} B_{1}^{2}\right)(\sin (w(\eta)))^{2} \cos (w(\eta)) \\
& +\left(a p A_{0}^{2} B_{1}-a \nu B_{1}\right) \sin (w(\eta)) \cos (w(\eta)) \\
& +\left(2 a^{3} q A_{1}-a p A_{1} B_{1}^{2}\right)(\sin (w(\eta)))^{4} \\
& +\left(-a p A_{0}^{2} A_{1}+a \nu A_{1}\right)(\sin (w(\eta)))^{2} \\
& -2(\sin (w(\eta)))^{3} a p A_{0} A_{1} B_{1}=0
\end{aligned}
$$

Some algebra and equating the coefficients of $\sin$ and cos functions to zero leads the following algebraic system:

$$
\begin{aligned}
-a A_{1}\left(6 a^{2} q+p A_{1}{ }^{2}-3 p B_{1}{ }^{2}\right)+8 a^{3} q A_{1}-a p A_{0}{ }^{2} A_{1}+a p A_{1}{ }^{3}-4 a p A_{1} B_{1}{ }^{2}+a \nu A_{1} & =0 \\
-2 a p A_{0} A_{1} B_{1} & =0 \\
6 a^{3} q B_{1}+3 a p A_{1}{ }^{2} B_{1}-a p B_{1}{ }^{3} & =0 \\
4 a p A_{0} A_{1} B_{1} & =0 \\
-5 a^{3} q B_{1}+a p A_{0}{ }^{2} B_{1}-2 a p A_{1}{ }^{2} B_{1}+a p B_{1}{ }^{3}-a \nu B_{1} & =0 \\
6 a^{3} q A_{1}+a p A_{1}{ }^{3}-3 a p A_{1} B_{1}{ }^{2} & =0 \\
2 a p A_{0}\left(A_{1}{ }^{2}-B_{1}{ }^{2}\right) & =0 \\
-2 a p A_{0}\left(A_{1}{ }^{2}-B_{1}{ }^{2}\right) & =0 \\
-14 a^{3} q A_{1}+a p A_{0}{ }^{2} A_{1}-2 a p A_{1}{ }^{3}+7 a p A_{1} B_{1}{ }^{2}-a \nu A_{1}+a A_{1}\left(6 a^{2} q+p A_{1}{ }^{2}-3 p B_{1}{ }^{2}\right) & =0
\end{aligned}
$$


Solving this system for $a, \nu, A_{0}, A_{1}$ and $B_{1}$ gives the solution set

$$
\begin{array}{llll}
\nu=a^{2} q, & A_{0}=0, A_{1}=0, & B_{1}=\sqrt{\frac{6 q}{p}} a \\
\nu=a^{2} q, & A_{0}=0, A_{1}=0, & B_{1}=-\sqrt{\frac{6 q}{p}} a \\
\nu=-a^{2} q, & A_{0}=0, A_{1}=\sqrt{-\frac{6 q}{p}} a, & B_{1}=0 \\
\nu=-a^{2} q, & A_{0}=0, A_{1}=-\sqrt{-\frac{6 q}{p}} a, & B_{1}=0 \\
\nu=-a^{2} q / 2, & A_{0}=0, A_{1}=\sqrt{-\frac{3 q}{2 p}} a, & B_{1}=\sqrt{\frac{3 q}{2 p}} a \\
\nu=-a^{2} q / 2, & A_{0}=0, A_{1}=\sqrt{-\frac{3 q}{2 p}} a, & B_{1}=-\sqrt{\frac{3 q}{2 p}} a \\
\nu=-a^{2} q / 2, & A_{0}=0, A_{1}=-\sqrt{-\frac{3 q}{2 p}} a, & B_{1}=\sqrt{\frac{3 q}{2 p}} a \\
\nu=-a^{2} q / 2, & A_{0}=0, A_{1}=-\sqrt{-\frac{3 q}{2 p}} a, & B_{1}=-\sqrt{\frac{3 q}{2 p}} a
\end{array}
$$

for arbitrary constant $a$. Thus, the solutions in both real and complex forms are constructed as

$$
\begin{aligned}
u_{4,5}(z, t) & = \pm \sqrt{\frac{6 q}{p}} a \operatorname{sech} a\left(z-a^{2} q \frac{t^{\gamma}}{\gamma}\right) \\
u_{6,7}(z, t) & = \pm \sqrt{\frac{-6 q}{p}} a \tanh a\left(z+a^{2} q \frac{t^{\gamma}}{\gamma}\right) \\
u_{8,9}(z, t) & =\sqrt{\frac{-3 q}{2 p}} a \tanh a\left(z+\frac{a^{2} q}{2} \frac{t^{\gamma}}{\gamma}\right) \pm \sqrt{\frac{3 q}{2 p}} a \operatorname{sech} a\left(z+\frac{a^{2} q}{2} \frac{t^{\gamma}}{\gamma}\right) \\
u_{10,11}(z, t) & =-\sqrt{\frac{-3 q}{2 p}} a \tanh a\left(z+\frac{a^{2} q}{2} \frac{t^{\gamma}}{\gamma}\right) \pm \sqrt{\frac{3 q}{2 p}} a \operatorname{sech} a\left(z+\frac{a^{2} q}{2} \frac{t^{\gamma}}{\gamma}\right)
\end{aligned}
$$

for arbitrary $a$. A particular form of the solution $u_{6}(z, t)$ (the positive signed one) calculated by choosing the parameters $a=q=1$ and $p=-1$ is pictured out in Fig 2(a)-2(d) for various values of $\gamma$. The initial wave moves along $z$-axis without changing its initial shape and height as time proceeds. The velocity of propagation is not constant when $\gamma<1$. When $\gamma$ value approaches 1 , the propagation speed also gets closer to a constant. Finally, $\gamma=1$ allows the initial wave a uniform propagation with constant speed. 


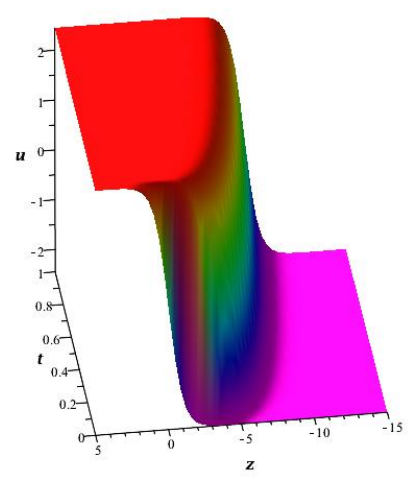

(a) $\gamma=0.25$

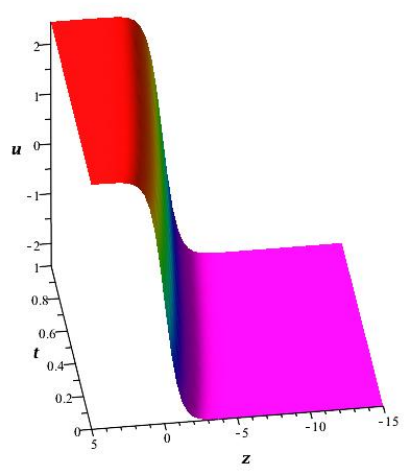

(c) $\gamma=0.75$

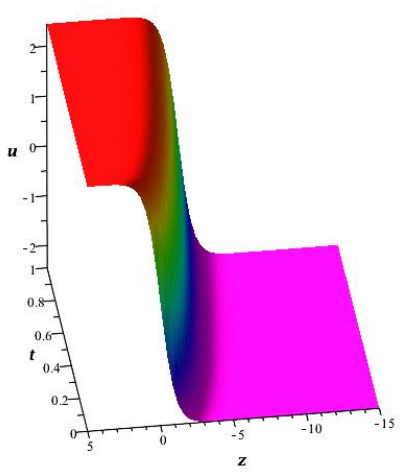

(b) $\gamma=0.50$

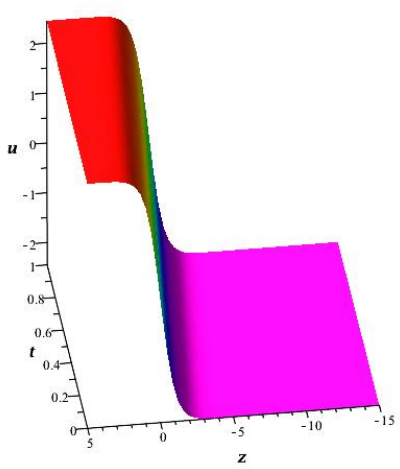

(d) $\gamma=1$

Figure 2: The solution $u_{6}(z, t)=\sqrt{6} \tanh \left(z+\frac{2 t^{\gamma}}{\gamma}\right)$ for various derivative orders

\section{Conclusion}

Fractional traveling wave transform succeeds both reducing the time fractional $\mathrm{KdV}$ and $\mathrm{mKdV}$ equations to some ODEs and giving the relation between trigonometric and hyperbolic functions based on time fractional Sine-Gordon equation. The predicted solution of a finite series form is constructed with aid of time fractional Sine-Gordon equation and homogeneous balance technique. The relation derived from time fractional Sine-Gordon equation provides the solutions to be expressed in multiplication of hyperbolic functions. Some real and complex valued solutions are calculated for both time fractional $\mathrm{KdV}$ and time fractional mKdV equations. The plots of some solutions are indicators of shape and height preserved initial wave propagations. 


\section{References}

[1] Boussinesq, J. (1877), Essai sur la theorie des eaux courantes, Memoires presentes par divers savants l'Acad. des Sci. Inst. Nat. France, XXIII, pp. 1-680.

[2] Korteweg, D. J., de Vries, G. (1895), "On the Change of Form of Long Waves Advancing in a Rectangular Canal, and on a New Type of Long Stationary Waves", Philosophical Magazine, 39 (240): 422-443.

[3] Miura, Robert M., Gardner, Clifford S., Kruskal, Martin D. (1968), "Korteweg-de Vries equation and generalizations. II. Existence of conservation laws and constants of motion", J. Mathematical Phys., 9 (8): 1204-1209.

[4] Wadati, M., \& Toda, M. (1972). The exact N-soliton solution of the Kortewegde Vries equation. Journal of the Physical Society of Japan, 32(5), 1403-1411.

[5] Hirota, R. (1971). Exact solution of the Korteweg-de Vries equation for multiple collisions of solitons. Physical Review Letters, 27(18), 1192.

[6] Wazzan, L. (2009). A modified tanh-coth method for solving the KdV and the KdV-Burgers' equations. Communications in Nonlinear Science and Numerical Simulation, 14(2), 443-450.

[7] Wang, M., Li, X., \& Zhang, J. (2008). The (G'/G)-expansion method and traveling wave solutions of nonlinear evolution equations in mathematical physics. Physics Letters A, 372(4), 417-423.

[8] Zheng-De, D., Zhen-Jiang, L., \& Dong-Long, L. (2008). Exact periodic solitary-wave solution for KdV equation. Chinese Physics Letters, 25(5), 1531.

[9] Lü, D., Cui, Y., Lü, C., \& Wei, C. (2010). Novel composite function solutions of the modified KdV equation. Applied Mathematics and Computation, 217(1), 283-288.

[10] Wazwaz, A. M. (2004). A sine-cosine method for handling nonlinear wave equations. Mathematical and Computer modeling, 40(5-6), 499-508.

[11] He, J. H., \& Wu, X. H. (2006). Exp-function method for nonlinear wave equations. Chaos, Solitons \& Fractals, 30(3), 700-708.

[12] Khalil, R., Al Horani, M., Yousef, A., \& Sababheh, M. (2014). A new definition of fractional derivative. Journal of Computational and Applied Mathematics, 264, 65-70. 
[13] Korkmaz, A., \& Hosseini, K. (2017). Exact solutions of a nonlinear conformable time-fractional parabolic equation with exponential nonlinearity using reliable methods. Optical and Quantum Electronics, 49(8), 278.

[14] Hosseini, K., \& Ansari, R. (2017). New exact solutions of nonlinear conformable time-fractional Boussinesq equations using the modified Kudryashov method. Waves in Random and Complex Media, 1-9.

[15] Korkmaz A., On The Wave Solutions of Conformable Fractional Evolution Equations, Commun. Fac. Sci. Univ. Ank. Series A1, 67(1) 68-79, 2018.

[16] Korkmaz A., Exact Solutions to $(3+1)$ Conformable Time Fractional Jimbo-Miwa,Zakharov-Kuznetsov and Modified Zakharov-Kuznetsov Equations, Communications in Theoretical Physics, 2017, 67(5), 479-482.

[17] Kaplan, M., \& Hosseini, K. (2018). Investigation of exact solutions for the Tzitzéica type equations in nonlinear optics. Optik-International Journal for Light and Electron Optics, 154, 393-397.

[18] Hosseini, K., Mayeli, P., \& Ansari, R. (2017). Bright and singular soliton solutions of the conformable time-fractional Klein-Gordon equations with different nonlinearities. Waves in Random and Complex Media, 1-9.

[19] Hosseini, K., Mayeli, P., \& Ansari, R. (2017). Modified Kudryashov method for solving the conformable time-fractional Klein-Gordon equations with quadratic and cubic nonlinearities. Optik-International Journal for Light and Electron Optics, 130, 737-742.

[20] Kaplan, M., Mayeli, P., \& Hosseini, K. (2017). Exact traveling wave solutions of the Wu-Zhang system describing $(1+1)$-dimensional dispersive long wave. Optical and Quantum Electronics, 49(12), 404.

[21] Tasbozan, O., Çenesiz, Y., \& Kurt, A. (2016). New solutions for conformable fractional Boussinesq and combined KdV-mKdV equations using Jacobi elliptic function expansion method. The European Physical Journal Plus, 131(7), 244.

[22] Çenesiz, Y., Tasbozan, O., \& Kurt, A. (2017). Functional Variable Method for conformable fractional modified KdV-ZK equation and Maccari system. Tbilisi Mathematical Journal, 10(1), 117-125.

[23] Atangana, A., Baleanu, D., \& Alsaedi, A. (2015). New properties of conformable derivative. Open Mathematics, 13(1), 1-10. 
[24] Abdeljawad, T. (2015). On conformable fractional calculus. Journal of computational and Applied Mathematics, 279, 57-66.

[25] Yan, C. (1996), A simple transformation for nonlinear waves, Physics Letters A, 224, 77-84. 